\title{
DEEP AND SHALLOW SLICE KNOTS IN 4-MANIFOLDS
}

\author{
MICHAEL R. KLUG AND BENJAMIN M. RUPPIK
}

(Communicated by Shelly Harvey)

\begin{abstract}
We consider slice disks for knots in the boundary of a smooth compact 4-manifold $X^{4}$. We call a knot $K \subset \partial X$ deep slice in $X$ if there is a smooth properly embedded 2-disk in $X$ with boundary $K$, but $K$ is not concordant to the unknot in a collar neighborhood $\partial X \times I$ of the boundary.

We point out how this concept relates to various well-known conjectures and give some criteria for the nonexistence of such deep slice knots. Then we show, using the Wall self-intersection invariant and a result of Rohlin, that every 4-manifold consisting of just one 0 - and a nonzero number of 2-handles always has a deep slice knot in the boundary.

We end by considering 4-manifolds where every knot in the boundary bounds an embedded disk in the interior. A generalization of the MurasugiTristram inequality is used to show that there does not exist a compact, oriented 4-manifold $V$ with spherical boundary such that every knot $K \subset S^{3}=$ $\partial V$ is slice in $V$ via a null-homologous disk.
\end{abstract}

\section{Overview}

The Smooth 4-Dimensional Poincaré Conjecture (SPC4) proposes that every closed smooth 4-manifold $\Sigma$ that is homotopy equivalent to $S^{4}$ is diffeomorphic to the standard $S^{4}$. By work of Freedman [Fre82, it is known that if $\Sigma$ is homotopy equivalent to $S^{4}$, then $\Sigma$ is in fact homeomorphic to $S^{4}$. In stark contrast to the SPC4, it might be the case that every compact smooth 4-manifold admits infinitely many distinct smooth structures. The existence of an exotic homotopy 4-sphere is equivalent to the existence of an exotic contractible compact manifold with $S^{3}$ boundary [Mil65, p. 113], henceforth called an exotic homotopy 4-ball.

One possible approach to proving that a proposed exotic homotopy 4 -ball $\mathcal{B}$ is in fact exotic is to find a knot $K \subset S^{3}=\partial \mathcal{B}$, such that there is a smooth properly embedded disk $D^{2} \hookrightarrow \mathcal{B}$, with $\partial D^{2}$ mapped to $K$, where $K$ is not smoothly slice in the usual sense in the standard 4-ball $B^{4}$. A knot is (topologically/smoothly) slice in $B^{4}$ if and only if it is null-concordant in $S^{3} \times I=S^{3} \times[0,1]$, i.e. there is a properly embedded (locally flat/smooth) cylinder $S^{1} \times I \hookrightarrow S^{3} \times I$ whose oriented boundary is $K \subset S^{3} \times\{0\}$ together with the unknot $U \subset S^{3} \times\{1\}$. Another way of thinking about this strategy is that we want to find a knot $K$ in $S^{3}=\partial \mathcal{B}$ that bounds a properly embedded smooth disk in $\mathcal{B}$ but does not bound any such disk that is contained in a collar $S^{3} \times I$ of the boundary of $\mathcal{B}$. In this case, to verify the sliceness of $K$, we have to go "deep" into $\mathcal{B}$.

Received by the editors October 3, 2020, and, in revised form, January 11, 2021.

2020 Mathematics Subject Classification. Primary 57K40; Secondary 57K10.

Key words and phrases. Concordance in general 4-manifolds, slice disks, Murasugi-Tristraminequality.

The authors were supported by the Max Planck Institute for Mathematics in Bonn. 


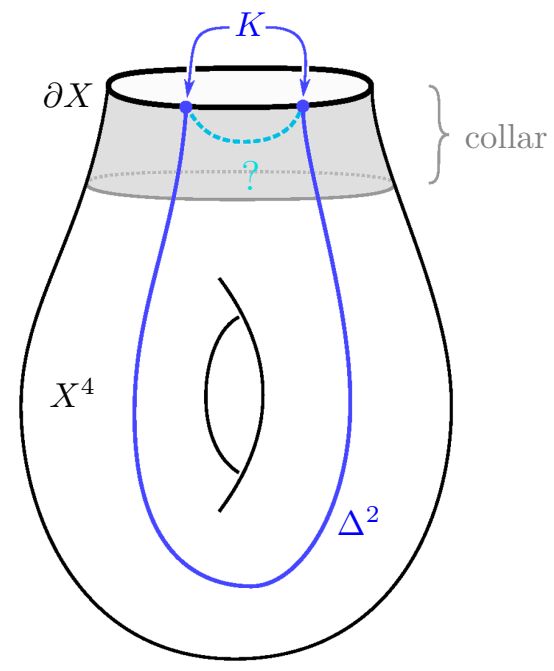

Figure 1. Schematic of a deep slice disk $\Delta^{2}$ (blue) in a 4-manifold $X^{4}$, with boundary the knot $K \subset \partial X$. The knot $K$ is called deep slice if it does not bound a properly embedded disk in a collar of the boundary, indicated by the (light blue) dashed lines.

An easier task might be to find a homology 4-ball $X$ with $S^{3}$ boundary such that there is a knot in the boundary that bounds a smooth properly embedded disk in $X$ but not in $B^{4}$, however, this is also an open problem. In FGMW10, the authors investigate the possibility of proving that a homotopy 4-ball $\mathcal{B}$ with $S^{3}$ boundary is exotic by taking a knot in the boundary that bounds a smooth properly embedded disk in $\mathcal{B}$ and computing the $s$-invariant of $K$, in the hopes that $s(K) \neq 0$, whereby they could then conclude that $\mathcal{B}$ is exotic. Unfortunately for this approach as noted in the paper, it turns out that the homotopy 4-ball that they were studying was in fact diffeomorphic to $B^{4}$, see $\mathrm{Akb10}$. It is still open whether the $s$-invariant can obstruct the sliceness of knots in $B^{4}$ that are slice in some homotopy 4-ball, as is noted in the corrigendum to KM13.

Motivated by this, we make the following definitions: For a 3-manifold $M^{3}$ containing a knot $K: S^{1} \hookrightarrow M$, we say that $K$ is null-concordant in $M \times I$ if there is a smoothly properly embedded annulus $S^{1} \times I \hookrightarrow M \times I$ cobounding $K \subset M \times\{0\}$ on one end and an unknot contained in a 3-ball $U \subset B^{3} \subset M \times\{1\}$ on the other. Equivalently, $K \subset M \times\{0\}$ bounds a smoothly properly embedded disk in $M \times I$.

Definition 1.1 (Deep slice/Shallow slice). Let $X^{4}$ be a smooth compact 4-manifold with nonempty boundary $\partial X$. We call a knot $K \subset \partial X$ slice in $X$ if there is a smooth properly embedded disk in $X$ with boundary $K$. We call a knot $K \subset \partial X$ shallow slice in $X$ if there is a smooth properly embedded disk in $\partial X \times I$ with boundary $K$ - this is equivalent to $K$ being null-concordant in the collar $\partial X \times I$. If $K$ is slice in $X$ but not shallow slice, we will call it deep slice in $X$. See Figure 1 for a schematic illustration of these definitions.

In this language, Problem 1.95 on Kirby's list Kir95 (attributed to Akbulut) can be reformulated as follows: Are there contractible smooth 4-manifolds with boundary an integral homology 3 -sphere which contain deep slice knots that are 
null-homotopic in the boundary? Note that any knot that is not nullhomotopic in the boundary will not be shallow slice and thus if it is slice, it will be deep slice. For this reason we will be looking for deep slice knots that are null-homotopic in the boundary. We will often consider our knots to be contained in 3-balls in the boundary, which we call local knots, so we can freely consider them in the boundary of any 4-manifold and discuss if they are slice there. To avoid confusion when we say that a (local) knot in a 3 -manifold $M^{3}$ is slice we will usually qualify it with "in $X^{4}$ ".

1.1. Outline. In the first part of this paper we will restrict ourselves to the smooth category, starting in section 2 where we discuss a condition that guarantees that some 4-manifolds have no deep slice knots and related results. In section 3, we prove that every 2-handlebody has a deep slice knot in its boundary. To do this we employ the Wall self-intersection number and a result of Rohlin which we discuss briefly.

Insection 4 we recall the Norman-Suzuki trick and observe that every 3-manifold bounds a 4-manifold where every knot in the boundary bounds a properly embedded disk. In contrast, if we restrict to slice disks trivial in relative second homology, we will see that every compact topological 4 -manifold with boundary $S^{3}$ contains a knot which does not bound a null-homologous topological slice disk. We finish with some questions and suggestions for further directions in section 5

1.2. Conventions. In the literature, properly embedded slice disks in a 4-manifold $X$ are often assumed to be null-homologous in $H_{2}(X, \partial X)$. We will make this extra assumption on homology only in section 4 when discussing the "universal slicings". For the first part deep slice and shallow slice will describe the existence of a embedded disks with the relevant properties without conditions on the homology class.

Starting from an $n$-manifold $M^{n}$ without boundary, we obtain a punctured $M$ (more precisely a bounded punctured $M$ ) by removing a small open $n$-ball $M^{\circ}:=$ $M \backslash$ int $D^{n}$, which yields a manifold with boundary $\partial M^{\circ}=S^{n-1}$. Observe that a punctured $M$ is the same as a connected sum $M^{\circ} \cong M \# D^{n}$ with a $n$-ball.

\section{Nonexistence OF DEEP SLICE KNOTS}

For starters, we have:

Proposition 2.1. There are no deep slice knots in $\natural^{k} S^{1} \times B^{3}$.

Proof. Let $K \subset \#^{k} S^{1} \times S^{2}=\partial\left(\natural^{k} S^{1} \times B^{3}\right)$ such that $K$ is slice in $\natural^{k} S^{1} \times B^{3}$. Then, thinking of $\natural^{k} S^{1} \times B^{3}$ as a wedge of $k$ copies of $S^{1}$ thickened to be 4-dimensional, if $D$ is any slice disk for $K$ we can isotope $D$ such that it does not intersect a onedimensional wedge of circles that $\natural^{k} S^{1} \times B^{3}$ deformation retracts onto. Therefore, $D$ can be isotoped to be contained in a collar neighborhood of the boundary $\#^{k} S^{1} \times S^{2}$ and thus $K$ is shallow slice.

The following might be a surprise, as one could expect that additional topology in a 3-manifold $M^{3}$ creates more room for concordances:

Proposition 2.2 (Special case of [NOPP19, Prop. 2.9]). If a local knot $K \subset B^{3} \subset$ $M^{3}$ is null concordant in $M^{3} \times I$, then $K$ is null concordant in $S^{3} \times I$. 
Proof sketch. Let $D$ be a properly embedded disk in $M \times I$ with boundary $K$ and let $\widetilde{M}$ be the universal cover of $M$. Then $D$ lifts to a properly embedded disk $\widetilde{D} \subset \widetilde{M} \times I$. Further, since $K$ is contained in a 3-ball $B$, all of the lifts of $K$ to $\widetilde{M}$ are just copies of $K$, and therefore, the boundary of $\widetilde{D}$ is a copy of $K$, considered inside of $\widetilde{M}$. As a consequence of geometrization Per03, we know that every universal cover of a punctured compact 3-manifold smoothly embeds into $S^{3}$, as was observed in [BN17, Lem. 2.11]. It follows then that there is an embedding $\widetilde{M} \times I \hookrightarrow S^{3} \times I$. But then the image of $\widetilde{D}$ under this embedding shows that $K$ bounds a disk in $S^{3} \times I$.

We have added a proof of this proposition here to highlight that this lifting argument breaks down in the case of higher genus surfaces if their inclusion induces a nontrivial map on fundamental groups. If $K$ bounds a genus $g$ surface with one boundary component $\Sigma_{g, 1}$ in $M \times I$, we can only lift this to the universal cover (and subsequently find a genus $g$ surface for $K$ in $S^{3}$ via this method) under the condition that the inclusion of $\Sigma_{g, 1}$ in $M \times I$ is $\pi_{1}$-trivial. So this argument does not work if the surface really "uses the extra topology of $M$ ".

Example 2.3. Take a non-orientable 3-manifold $M$ containing the connected sum $K \# K$ of two copies of a local invertible knot $K$ with smooth 4-ball genus $g^{4}(K \# K) \geq 2$. As an explicit example, $K$ a left-handed trefoil will work, and we illustrate the following in Figure 2. Describe an embedded torus in $M \times I$ with the motion picture method: Use the connected sum band to split the sum with a saddle. Then let one of the summands travel around an orientation reversing loop in $M$ while leaving the other one fixed. The summand traveling around the loop was reflected in the process and since it is invertible it is isotopic to $-K=r \bar{K}$ in a 3-ball neighborhood in $M$. Fusing the summands back together along a connected sum band we now obtain $K \#-K$ as a local knot. Finally cap this off with the usual ribbon disk for the connected sum of a knot with its concordance inverse. Therefore $g^{M \times I}(K \# K) \leq 1$ and by Proposition 2.2 in fact $g^{M \times I}(K \# K)=1$. The 4-ball genus $g^{4}(K \# K) \geq 2$ of this example is strictly larger than its $\left(M^{3} \times I\right)$-4-genus, which we define as

$$
\begin{aligned}
& g^{M \times I}(J):=\min \{g \mid \exists \text { smooth proper embedding } \\
& \left.\qquad \Sigma_{g, 1} \hookrightarrow M \times I \text { with } \partial \Sigma_{g, 1}=J \subset M \times\{0\}\right\}
\end{aligned}
$$

Observe that in this notation the usual 4-ball genus is $g^{4}=g^{S^{3} \times I}$ and we can rephrase Proposition 2.2 as $g^{M \times I}(K)=0$ implies $g^{4}(K)=0$ for local knots $K$. Similar notions of 4 -genera were introduced in Celoria's investigation of almostconcordance [Cel18, Def. 12].

It would be interesting to find an example of an orientable 3-manifold $M^{3}$ where the $g^{M \times I}(K)$ genus of some local knot $K \subset D^{3} \subset M$ is strictly smaller than the 4-ball genus $g^{4}(K)$, or prove that no such $M$ exists. Local $K$ satisfy $g^{M \times I}(K) \leq$ $g^{4}(K)$ as cobordisms in $S^{3} \times I$ can be embedded into $M \times I$. Because of Proposition 2.2 an example where these values differ can only appear for $g^{4}(K) \geq 2$. Moreover, as we will see in Proposition 2.4 such an $M$ would necessarily not embed in $S^{4}$. Another special case is treated in [DNPR18, Thm. 2.5] where a handle cancellation argument shows that there is no difference for local knots in $M=S^{1} \times S^{2}$, that is 

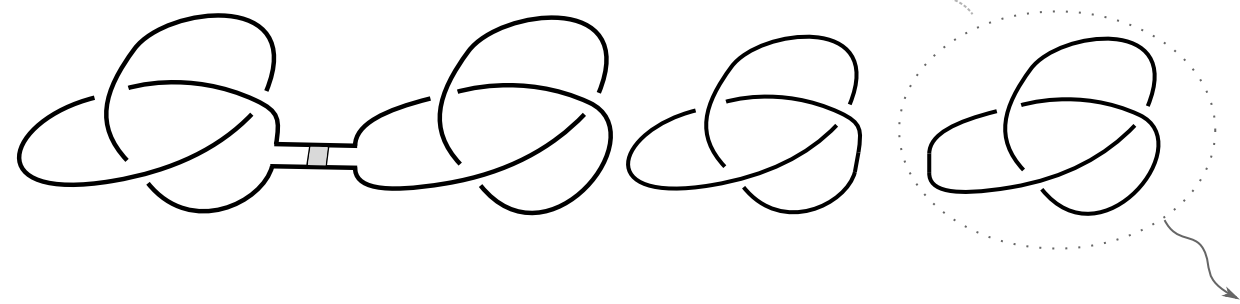

(А) Saddle move to separate the summands (в) One of the summands travels around an of $K \# K$.

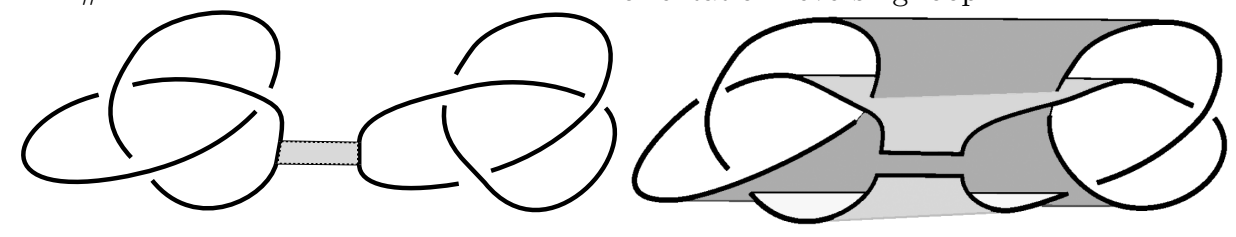

(C) It returns mirrored, now add a fusion (D) Finish off the movie with the standard band. ribbon disk for $K \#-K$.

Figure 2. Four frames of the movie of a properly embedded punctured torus in $M^{3} \times I$ with boundary $K \# K \subset M \times\{0\}$, where $M$ is a non-orientable 3-manifold.

the equality $g^{S^{1} \times S^{2} \times I}=g^{4}$ holds (and also analogous statements for $\#^{k} S^{1} \times S^{2}$ ). Topological concordance in $S^{1} \times S^{2} \times I$ is investigated in FNOP19.

We now give a criterion that shows that certain 4-manifolds have no local deep slice knots in the boundary. This idea is also contained in [Suz69, Thm. 0] and its variants.

Proposition 2.4. Let $X^{4}$ be a compact 4-manifold with a local knot $\gamma \subset B^{3} \subset \partial X$ that is slice in $X$. If there is a cover of $X$ which can be smoothly embedded into $S^{4}$, then $\gamma \subset B^{3} \hookrightarrow S^{3}=\partial B^{4}$ is slice in $B^{4}$. Hence, $\gamma$ is shallow slice in $X$.

Proof. Let $\widetilde{X}$ be a cover of $X$ with an embedding $\widetilde{X} \subset S^{4}$ into $S^{4}$ and let $\widetilde{D}$ be a lift of a slice disk for $\gamma$ to $\widetilde{X}$ with $\widetilde{\gamma}=\partial \widetilde{D}$. Note that the knot $\widetilde{\gamma}$ is the same as $\gamma$, since $\gamma$ is contained in a 3-ball and the only covers of a 3-ball are disjoint unions of 3-balls. Puncture $S^{4}$ by removing a small ball $B$ close to $\widetilde{\gamma}$ and such that $\widetilde{\gamma}$ can be connected by an annulus disjoint from $\tilde{X}$ to $\partial B$ and such that the other end of the annulus is (the mirror image of) $K \subset \partial B$. Then since $S^{4}-\operatorname{int} B \cong B^{4}$, the annulus together with $\widetilde{D}$ show that $K$ is slice in the $B^{4}$ which is the complement of the small ball. Therefore $\gamma$ is shallow slice in $X$.

As an example, Proposition 2.4 implies that $\natural^{k} S^{2} \times D^{2}$ contains no deep slice local knots, since these manifolds can all be embedded in $S^{4}$. However, these manifolds all contain deep slice knots, necessarily non-local, as will be seen shortly. Additionally, we have:

Corollary 2.5. Suppose that $X$ is a closed smooth 4-manifold with universal cover $\mathbb{R}^{4}$ or $S^{4}$, and let $X^{\circ}$ denote the punctured version. Then $X^{\circ}$ has no deep slice knots. 


\section{EXISTENCE OF DEEP SLICE KNOTS}

A 2-handlebody is a 4-manifold whose handle decomposition contains one 0handle, some nonzero number of 2-handles and no handles of any other index. Examples of this are knot traces, where a single 2-handle is attached along a framed knot to the 4-ball. In this section, we prove:

Theorem 3.1. Every 2-handlebody X contains a null-homotopic deep slice knot in its boundary.

Remark 3.2. For the special case of the 2-handlebody $D^{2} \times S^{2}$ the existence of such knots was already observed in [DNPR18, Thm. B] (here only winding number $w=0$ gives null homotopic knots). Furthermore the authors construct an infinite family of slice knots which are pairwise different in topological concordance in a collar of the boundary.

Theorem 3.1 breaks up naturally into two cases depending on whether the boundary has nontrivial $\pi_{1}$ or not (i.e. if it is or is not $\left.S^{3}\right)$. In the case where $\pi_{1}(\partial X) \neq 1$, there is a concordance invariant for knots in arbitrary 3-manifolds, closely related to the Wall self-intersection number (see [Wa199], FQ90, and [Sch03]), that will allow us to show that some obviously slice knots are not shallow slice. In the case where $\pi_{1}(\partial X)$ is trivial, and therefore by the 3-dimensional Poincaré conjecture Per03. $\partial X=S^{3}$, the Wall self-intersection number is of no use. However, in this case, the consideration of whether a knot that is slice in $X$ is deep slice in $X$ is related to the existence of spheres representing various homology classes in the manifold obtained by closing $X$ off with a 4 -handle.

Remark 3.3. If there was a direct proof that every closed homotopy 3-sphere smoothly bounds a contractible 4-manifold, then we would not need to invoke the 3-dimensional Poincaré conjecture.

Following Yil18 and Sch03, we briefly introduce the Wall self-intersection number in the setting that we will be working in, and state some of its basic properties. Let $Y^{3}$ be a closed oriented 3-manifold and let $\gamma: S^{1} \hookrightarrow Y$ be a knot in $Y$. Let $\mathcal{C}_{\gamma}(Y)$ denote the set of concordance classes of oriented knots in $Y$ that are freelyhomotopic to $\gamma$. In particular $\mathcal{C}_{U}(Y)$ denotes the set of concordance classes of oriented null-homotopic knots in $Y$, where we write $U$ for the local unknot in $Y$. Given an oriented null-homotopic knot $K \subset Y$, by transversality there exists an oriented immersed disk $D$ in $Y \times I$ with boundary $K \subset Y \times\{0\}=Y$ that has only double points of self-intersection. Let $\star \in Y$ denote a basepoint which we implicitly use for $\pi_{1}(Y)=\pi_{1}(Y \times I)$ throughout. Choose an arc, which we will call a whisker, from $\star$ to $D$. For each double point of self-intersection $p \in D$ choose a numbering of the two sheets of $D$ that intersect at $p$. Then let $g_{p} \in \pi_{1}(Y)$ be the homotopy class of the loop in $Y \times I$ obtained by starting at $\star$, taking the whisker to $D$, taking a path to $p$ going in on the first sheet, taking a path back to where the whisker meets $D$ that leaves $p$ on the second sheet, and then returning to $\star$ using the whisker. Note that changing the order of the two sheets would transform $g_{p}$ to $g_{p}^{-1}$. Also, since $K$ and $Y$ are oriented, $D$ and $Y \times I$ obtain orientations with the convention that $K \subset Y \times\{0\}=Y$, and therefore, for every self-intersection point $p \in D$, there is an associated sign which we will denote by $\operatorname{sign}(p)$.

Let

$$
\widetilde{\Lambda}:=\frac{\mathbb{Z}\left[\pi_{1}(Y)\right]}{\left\langle\left\{g-g^{-1} \mid g \in \pi_{1}(Y)\right\}\right\rangle \oplus \mathbb{Z}[1]}
$$




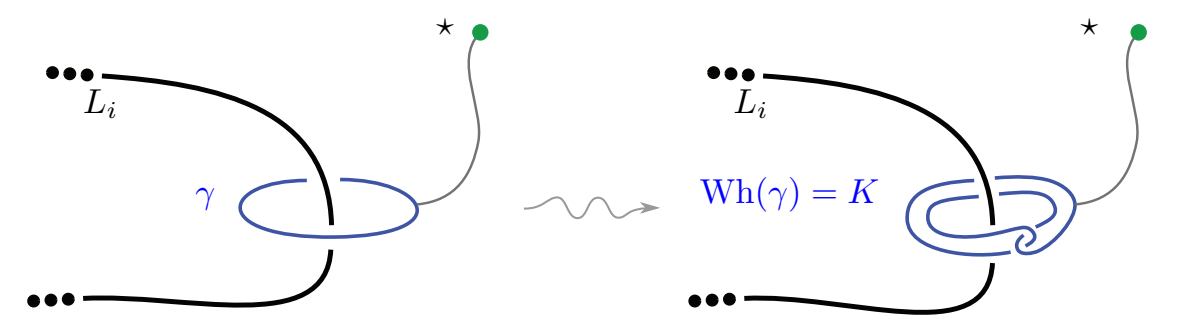

Figure 3. The Whitehead double of a nontrivial meridian $\gamma$ to one of the surgery link components is deeply slice in $X$.

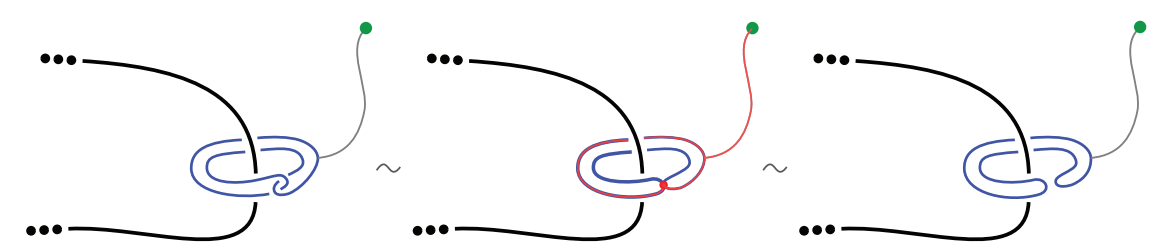

FiguRE 4. Track of a homotopy from the Whitehead double of $\gamma$ to the unknot giving an immersed disk with a single double point (red in the middle frame). The red double point loop based at the green basepoint calculates that $\mu(K)=\gamma$.

were the quotient is a quotient as abelian groups. The Wall self-intersection number of $K$ is defined to be

$$
\mu(K)=\sum_{p} \operatorname{sign}(p) \cdot g_{p} \in \widetilde{\Lambda}
$$

See [Sch03] for a proof that it is independent of the choice of $D$, the choice of whisker, and the choice of orderings of the sheets of $D$ around the double points. Further, $\mu$ is a concordance invariant in $Y \times I$, and therefore defines a map:

$$
\mu: \mathcal{C}_{U}(Y) \rightarrow \widetilde{\Lambda}
$$

Notice that if $g \in \pi_{1}(Y)$ and $g \neq 1$ then $g$ is also nonzero in $\widetilde{\Lambda}$.

Proof of Theorem 3.1, Case 1. We are now in position to handle Theorem 3.1 in the case where $\pi_{1}(\partial X) \neq 1$. Now $X$ is described by attaching 2 -handles to $D^{4}$ along some framed link $L \subset \partial D^{4}$. Since $\pi_{1}(\partial X)$ is (normally) generated by the meridians of $L$ and $\pi_{1}(\partial X) \neq 1$, there is some meridian $\gamma$ of $L$ that is nontrivial in $\pi_{1}(\partial X)$. Notice that if we are given a 2-handlebody described by a framed link $L$ and $K$ is a knot in the boundary of the 2-handlebody that is shown in the framed link diagram as an unknot (possibly linked with $L$ ), then $K$ is slice in the 2-handlebody - just forget all of the other 2-handles and take an unknotting disk whose interior has been pushed into the 0 -handle. Now, take $K$ in $\partial X$ to be a Whitehead double of $\gamma$ as in Figure 3, which is a null-homotopic knot in the boundary. By the previous observation, since $K$ is unknotted in the boundary of the 0 -handle, $K$ is slice in $X$. Additionally, one computes that $\mu(K)=\gamma \neq 1 \in \widetilde{\Lambda}$, for example using the null-homotopy in Figure 4. Therefore, $K$ is not null-concordant in $\partial X$, so $K$ is deep slice in $X$. 
Notice that if $\pi_{1}(\partial X)=1$, then $\mu$ is of no use since $\tilde{\Lambda}=0$. Now assume that $\pi_{1}(\partial X)=1$ so that $\partial X=S^{3}$. Again $X$ is obtained by attaching 2-handles to some framed link $L$. Let $\widehat{X}$ denote the closed 4-manifold obtained by closing off $X$ with a 4-handle. We will need a lemma on surfaces in 2-handlebodies, whose statement is standard and could alternatively be concluded from the KSS-normal form for surfaces as in [Kam17, Thm. 3.2.7] and KSS82.

Lemma 3.4. Let $X$ be a closed smooth 4-manifold with a handle decomposition consisting of only 0-, 2-, and 4-handles, with exactly one 0-handle and one 4-handle. Every element of $H_{2}(X ; \mathbb{Z})$ can be represented by a smooth closed orientable surface whose intersection with the union of the 0 - and 2-handles of $X$ is a single disk.

Proof. Let $X_{\leq 2}$ denote the union of the 0 - and 2-handles of $X$, so that $X=$ $X_{\leq 2} \cup B^{4}$. For every 2 -handle $h_{i}$, there is an element $H_{2}(X ; \mathbb{Z})$ obtained by taking the co-core disk $D_{i}$ for $h_{i}$ and capping it off with an orientable surface in the 4handle. Let $\left\{F_{i}\right\}$ denote a choice of these surfaces, one for each 2-handle. These surfaces form a basis for $H_{2}(X ; \mathbb{Z})$ and note that each has the desired property that $F_{i} \cap X_{\leq 2}=D_{i}$ is a disk.

Given an arbitrary element $x \in H_{2}(X ; \mathbb{Z})$, we have $x=a_{1}\left[F_{1}\right]+\cdots+a_{n}\left[F_{n}\right]$ for some $a_{i} \in \mathbb{Z}$. Therefore, by taking parallel copies of the $F_{i}$ for each summand, we can find an immersed (possibly disconnected) orientable surface $F^{\prime}$ representing $x$, with $F^{\prime} \cap X_{\leq 2}$ a union of $\sum\left|a_{i}\right|$ disjoint disks. By taking arcs in $\partial X_{\leq 2}$ that connect the different boundaries of the disks all together, and attaching tubes to $F^{\prime}$ along these arcs, we obtain a connected orientable immersed surface $F^{\prime \prime}$ representing $x$ whose intersection with $X_{\leq 2}$ is now a disk. In particular, the tubing is done so that half of the tube is contained in $X_{\leq 2}$ and the other half is in the 4-handle, and therefore $F^{\prime \prime} \cap X_{\leq 2}$ is the result of boundary summing together the disks in $F^{\prime} \cap X_{\leq 2}$.

To make $F^{\prime \prime}$ into an embedded surface, we can resolve the double points in the 4-handle, by increasing the genus, and arrive at a surface representing $x$ with the desired property.

The main ingredient for the proof of the second case of Theorem 3.1 is the following theorem of Rohlin, and in particular the corollary that follows. Rohlin's theorem has been used in a similar way to study slice knots in punctured connected sums of projective spaces, for example in YYas91 and Yas92.

Theorem 3.5 (Rohlin, Roh71]). Let $X$ be an oriented closed smooth 4-manifold with $H_{1}(X ; \mathbb{Z})=0$. Let $\psi \in H_{2}(X ; \mathbb{Z})$ be an element that is divisible by 2 , and let $F$ be a closed oriented surface of genus $g$ smoothly embedded in $X$ that represents $\psi$. Then

$$
4 g \geq|\psi \cdot \psi-2 \sigma(X)|-2 b_{2}(X)
$$

Corollary 3.6. Let $X$ be a closed smooth 4-manifold with $H_{1}(X ; \mathbb{Z})=0$, and $H_{2}(X ; \mathbb{Z}) \neq 0$. Then there exists a homology class $\psi \in H_{2}(X ; \mathbb{Z})$ that cannot be represented by a smoothly embedded sphere.

Proof of Corollary 3.6. To apply Theorem 3.5 we must find a homology class $\psi$ that is divisible by 2 where the right hand side $|\psi \cdot \psi-2 \sigma(X)|-2 b_{2}(X)>0$. Since the intersection form on $X$ is unimodular, there exists some element $\alpha$ with $\alpha \cdot \alpha \neq 0$. From Poincaré duality together with the universal coefficient theorem and 


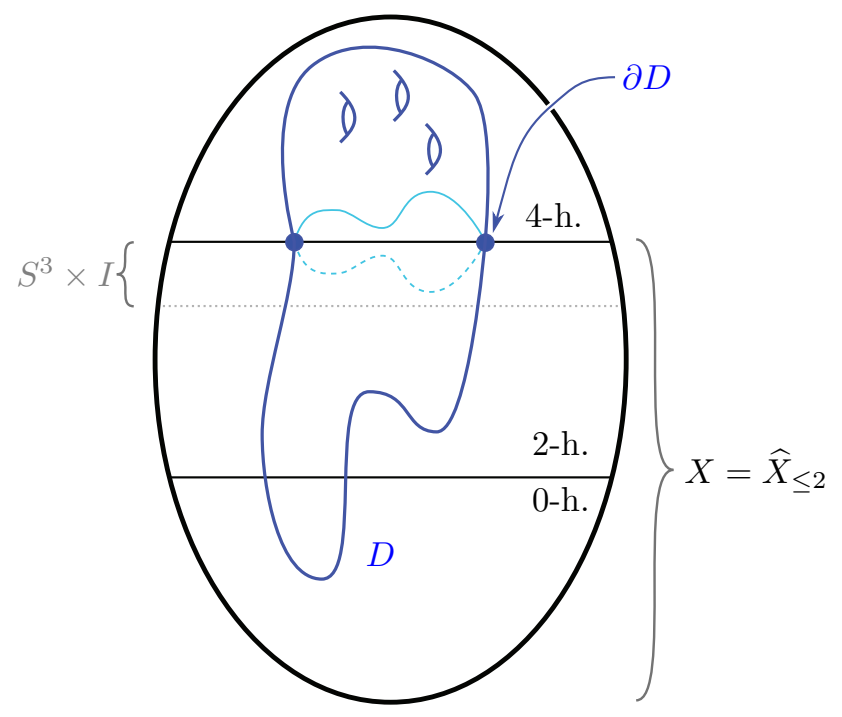

Figure 5. Schematic of the blue surface $F$ in the 2-handlebody, intersecting $X=$ the union of the 0 - and 2-handles in a disk $D$. If $\partial D$ was shallow slice (dashed light blue) in $X$, disk $D$ union the shallow slice disk flipped into the 4-handle (solid light blue) would be an impossible sphere representative of the homology class of $F$.

our hypothesis that $H_{1}(X ; \mathbb{Z})=0$, we know that $H_{2}(X ; \mathbb{Z})$ is torsion free. Then by taking $k$ to be a sufficiently large integer, we can make $|(2 k \alpha) \cdot(2 k \alpha)-2 \sigma(X)|$ arbitrarily large. By taking $\psi=2 k \alpha$, the result follows.

Proof of Theorem 3.1, Case 2. By Corollary 3.6 let $\psi \in H_{2}(\widehat{X} ; \mathbb{Z})$ be a homology class that can not be represented by an embedded sphere. Using Lemma 3.4 let $F$ be a smooth closed orientable surface representing $\psi$ whose intersection with $X=\widehat{X}_{\leq 2}$ is a disk $D$, as illustrated schematically in Figure 5. Then $\partial D \subset \partial X$ is deep slice in $X$, since otherwise the surface obtained by intersecting $F$ with the 4-handle could be replaced with a disk without altering the homology class, violating the assumption that $\psi$ cannot be represented by an embedded sphere. To see that the homology class is not altered, observe that in any 2-handlebody the homology class of a surface is determined by how it intersects the 0 - and 2-handles. Also observe that in this case the deep slice knot $\partial D \subset \partial X=S^{3}$ is local. This concludes the proof of Theorem 3.1

\section{UNIVERSAL SLICING MANIFOLDS DO NOT EXIST}

The Norman-Suzuki trick [Nor69, Cor. 3], Suz69, Thm. 1] can be used to show that any knot $K \subset S^{3}$ bounds a properly embedded disk in a punctured $S^{2} \times S^{2}$ : The track of a null-homotopy of $K$ in $D^{4}$ can be placed in the punctured $S^{2} \times S^{2}$ which gives a disk that we can assume to be a generic immersion, missing $S^{2} \vee S^{2} \subset$ $\left(S^{2} \times S^{2}\right)^{\circ}$, and with a finite number of double points. By tubing into the spheres $S^{2} \times\{\mathrm{pt}\},\{\mathrm{pt}\} \times S^{2}$ we can remove all the intersections - but observe that this changes the homology class of the disk. 
Proposition 4.1. Let $M^{3}$ be a closed orientable 3-manifold. There exists a compact orientable 4-manifold $X^{4}$ constructed with only a 0-handle and 2-handles, with $\partial X=M$ such that every knot in $M$ is slice in $X$.

Proof. Start by taking any compact 4-manifold $X^{\prime}$ with only 0-, 2-handles and boundary $M$ and let $X=X^{\prime} \#\left(S^{2} \times S^{2}\right)$. Let $K \subset M=\partial X$ be a knot. Since $X^{\prime}$ and $X$ are simply connected, $K$ bounds an immersed disk which we can assume lives completely in the $X^{\prime}$-summand of the connected sum. Now the NormanSuzuki trick works to remove intersection points of the immersion by tubing into the coordinate spheres of the $S^{2} \times S^{2}$-summand.

Remark 4.2. In contrast to the homologically nontrivial disks constructed in the Norman-Suzuki trick, a knot is slice via a null-homologous disk in some connected sum $\#^{n} S^{2} \times S^{2}$ if and only if its Arf-invariant is zero. Arf $K=0$ implies that the knot is band-pass equivalent to the unknot, and a band pass can be realized by sliding the (oppositely oriented) strands of a pair of bands over the coordinate spheres in a $S^{2} \times S^{2}$ factor. Conway-Nagel [CN20] defined and studied the minimal number of summands needed to find a disk in a punctured $\#^{n} S^{2} \times S^{2}$.

Convention. From now until the end of this section, properly embedded slice disks $\Delta^{2} \subset X^{4}$ in a 4-manifold are always required to be null-homologous. We will still add the qualifier "null-homologous" in the statements to emphasize this. Since our obstructions work in the topologically locally flat category, we will formulate everything in this more general setting.

Definition 4.3. A knot $K \subset S^{3}$ is (topologically/smoothly) null-homologous slice in the (topological/smooth) 4-manifold $X^{4}$ with $\partial X=S^{3}$, if $K=\partial \Delta$, where $\Delta^{2} \subset X$ is a (locally flat/smooth) properly embedded disk such that $[\Delta, \partial \Delta]=0 \in$ $H_{2}(X, \partial X)$.

One way of studying if a knot $K$ is slice in $D^{4}$ is to approximate $D^{4}$ by varying the 4-manifold $X$. By restricting the intersection form and looking at simply-connected 4-manifolds $X$ this gives rise to various filtrations of the knot concordance group (notably the $(n)$-solvable filtration $\mathcal{F}_{n}$ of Cochran-Orr-Teichner COT03 and the positive and negative variants $\mathcal{P}_{n}, \mathcal{N}_{n}$ [CHH13]).

We say that the properly embedded disk $\Delta$ is null-homologous if its fundamental class $[\Delta, \partial \Delta] \in H_{2}(X, \partial X)$ is zero. Since by Poincaré duality the intersection pairing $H_{2}(X) \otimes_{\mathbb{Z}} H_{2}(X, \partial X) \stackrel{\pitchfork}{\rightarrow} \mathbb{Z}$ is non-degenerate, a null-homologous disk is characterized by the property that it intersects all closed second homology classes algebraically zero times. For slicing in arbitrary 4-manifolds, we here restrict to null-homologous disks to exclude constructions as in the Norman-Suzuki trick.

For every fixed knot $K \subset S^{3}$, there is a 4-manifold in which $K$ is null-homologically slice. Norman [Nor69, Thm. 4] already observes that it is possible to take as the 4-manifold a punctured connected sum of the twisted 2 -sphere bundles $S^{2} \widetilde{\times} S^{2}$. Similarly, CL86, Lem. 3.4] discuss that for any knot $K \subset S^{3}$ there are numbers $p, q \in \mathbb{N}$ such that $K$ is null-homologous slice in the punctured connected sum $\#^{p} \mathbb{C P}^{2} \# \overline{\mathbb{C P}^{2}}$ of complex projective planes. The argument starts with a sequence of positive and negative crossing changes leading from $K$ to the unknot, and then realizes say a positive crossing change by sliding a pair of oppositely oriented strands over the $\mathbb{C P}^{1}$ in a projective plane summand. The track of this isotopy, together with a disk bounding the final unknot gives a motion picture of a null-homologous slice 
disk. Since both positive and negative crossing changes might be necessary, it is important that both orientations $\mathbb{C P}^{2}, \overline{\mathbb{C P}^{2}}$ are allowed to appear in the connected sum.

In view of $\left(S^{2} \times S^{2}\right)^{\circ}$ where every knot in the boundary bounds a disk (which is rarely null-homologous) and $\left(\#^{p} \mathbb{C P}^{2} \#^{q} \overline{\mathbb{C P}^{2}}\right)^{\circ}$, in which we find plenty of nullhomologous disks (but only know how many summands $p, q$ we need after fixing a knot on the boundary) a natural question concerns the existence of a universal slicing manifold. Is there a fixed compact, smooth, oriented 4-manifold $V^{4}$ with $\partial V=S^{3}$ such that any knot $K \subset S^{3}$ is slice in $V$ via a null-homologous disk? It turns out that a signature estimate shows such a universal solution cannot exist.

Theorem 4.4. Any compact oriented 4-manifold $V^{4}$ with $\partial V=S^{3}$ contains a knot in its boundary that is not topologically null-homologous slice in $V$.

Remark 4.5. If we drop the assumption that $V$ should be compact, a punctured infinite connected sum of projective planes does the job:

$$
D^{4} \#^{\infty}\left(\mathbb{C P}^{2} \# \overline{\mathbb{C P}^{2}}\right)
$$

For any fixed knot on the boundary there is a compact slice disk in a finite stage

$$
D^{4} \#^{k} \mathbb{C P}^{2} \#^{l} \overline{\mathbb{C P}^{2}} \# D^{4} \subset D^{4} \#^{\infty}\left(\mathbb{C P}^{2} \# \overline{\mathbb{C P}^{2}}\right) \text {. }
$$

The remainder of this section is concerned with a proof of Theorem 4.4. As preparation, let us specialize a result CN20, Thm. 3.8], which is a generalization of the Murasugi-Tristram inequality for links bounding surfaces in 4-manifolds, to the case of knots. Here $\sigma_{\omega}(K)$ is the Levine-Tristram signature of the knot $K$, defined as the signature of the hermitian matrix $(1-\omega) V+(1-\bar{\omega}) V^{T}$, where $V$ is a Seifert matrix of $K$ and $\omega$ a unit complex number not equal to 1 . References for this signature include Lev69, [Tri69] and the recent survey Con19]. The following inequality only holds for specific values of $\omega$, and will adopt the notation $S_{!}^{1}$ for unit complex numbers $\omega \in S^{1}-\{1\}$ which do not appear as a zero of an integral Laurent polynomial $p \in \mathbb{Z}\left[t, t^{-1}\right]$ with $p(1)= \pm 1$.

Theorem 4.6 ([CN20, Special case of Thm. 3.8]). Let $X$ be a closed oriented topological 4-manifold with $H_{1}(X ; \mathbb{Z})=0$. If $\Sigma \subset\left(S^{3} \times I\right) \# X$ is a null-homologous (topological) cobordism between two knots $K \subset S^{3} \times\{0\}$ and $-K^{\prime} \subset-\left(S^{3} \times\{1\}\right)$, each contained in one of the two boundary component $S^{3}$ 's of $\left(S^{3} \times I\right) \# X$, then

$$
\left|\sigma_{K^{\prime}}(\omega)-\sigma_{K}(\omega)+\operatorname{sign}(X)\right|-\chi(X)+2 \leq-\chi(\Sigma)
$$

for all $\omega \in S_{!}^{1}$.

For $K \subset \partial X^{\circ}$ which is null-homologous slice in $X$ and $\Sigma$ an annulus, we can further simplify:

Corollary 4.7. Let $X$ be a closed topological 4-manifold with $H_{1}(X ; \mathbb{Z})=0$. If the knot $K \subset S^{3}$ is topologically null-homologous slice in $X^{\circ}$ then for $\omega \in S_{!}^{1}$ we have

$$
\left|\sigma_{K}(\omega)+\operatorname{sign}(X)\right|-\chi(X)+2 \leq 0
$$

To prove Theorem 4.4 it will be enough to obstruct the sliceness of a single knot in the boundary. The strategy is to use surgery to trivialize $H_{1}$, then pick the knot $K$ in the original manifold boundary and arrive at a contradiction to Corollary 4.7 in the surgered manifold if $K$ was null-homologous slice. 
Proof of Theorem 4.4. Let $V$ be a compact topological 4-manifold with boundary $S^{3}$, we want to find a knot in its boundary which is not slice. Pick a set of disjointly embedded loops $\gamma_{1}, \ldots, \gamma_{l}$ in $V$ whose homology classes generate $H_{1}(V)$. If $V$ already satisfies $H_{1}(V)=0$, set $l=0$ for the remainder of the proof and omit the surgery altogether. Let $K$ be a knot in $S^{3}$ whose signature (at the unit complex number $\omega=-1$ ) satisfies

$$
\left|\sigma_{K}(-1)\right| \geq|\operatorname{sign}(V)|+|\chi(V)|+2 l .
$$

Note that the constant on the right hand side only depends on the signature, Euler characteristic, and number of generators of $H_{1}(V)$, and not on the knot $K$. For example, since signature is additive under connected sum, the self-sum $K_{n}=\#^{n} K$ with $n$ large enough has arbitrarily high signature at $\omega=-1$ if we start with a $K$ that has positive signature $\sigma_{K}(-1)$ (for example, taking $K$ to be the left-handed trefoil knot).

Suppose that $K$ is slice in $V$ via a null-homologous disk $\Delta$. Being null-homologous in the relative second homology group means geometrically that there is a locally flat embedded 3-manifold $M^{3}$ with boundary the slice disk $\Delta$ union a Seifert surface for $K$ in the boundary $S^{3}$, see [Lic97, Lem. 8.14]. We can remove the closed components from $M$, what remains is a 3-manifold with nonempty boundary in $V$. Generically the embedded circles $\gamma_{1}, \ldots, \gamma_{l}$ will intersect the 3 -manifold $M$ in points, but we can push these intersection points off the boundary of $M$ via an isotopy of the curves in $V$. We will still keep the notation $\gamma_{1}, \ldots, \gamma_{l}$ for the isotoped curves which are now disjoint from $M$. Essentially, this finger move supported in a neighborhood of $M$ is guided by pairwise disjoint arcs in $M$ connecting the intersections points to the boundary.

Perform surgery on the loops $\gamma_{1}, \ldots, \gamma_{l}$, i.e. for each $\gamma_{i}$ remove an open tubular neighborhood $\nu\left(\gamma_{i}\right) \cong S^{1} \times \operatorname{int} D^{3}$ and glue copies of $D^{2} \times S^{2}$ to the new $S^{1} \times S^{2}$ boundary components via the identity map $S^{1} \times S^{2} \rightarrow S^{1} \times S^{2}$. After this surgery we have a compact 4-manifold $V^{\prime}$ with $H_{1}\left(V^{\prime}\right)=0$, and the original disk $\Delta$ survives into $V^{\prime}$ in which we will call it $\Delta^{\prime}$. Observe that this "new" disk $\Delta^{\prime}$ is still nullhomologous in $V^{\prime}$, since the 3-manifold is still present after the surgery. Each circle surgery in a 4-manifold increases the Euler characteristic by 2, thus $\chi\left(V^{\prime}\right)=$ $\chi(V)+2 l$. By construction, the 4-manifolds $V$ and $V^{\prime}$ are cobordant, and so their signatures $\operatorname{sign}\left(V^{\prime}\right)=\operatorname{sign}(V)$ agree.

Starting with a knot $K$ with large enough signature, if there existed a nullhomologous $\Delta^{\prime}$, since $H_{1}\left(V^{\prime}\right)=0$ :

$$
\left|\sigma_{K}(-1)+\operatorname{sign}\left(V^{\prime}\right)\right|-\chi\left(V^{\prime}\right)+2=\left|\sigma_{K}(-1)+\operatorname{sign}(V)\right|-(|\chi(V)|+2 l)+2>0
$$

which contradicts the inequality in Corollary 4.7. Therefore $\Delta$ for $K$ cannot exist.

Remark 4.8. Earlier sources for results in the smooth category include Gilmer and Viro's Gil81 version of the Murasugi-Tristram inequality for the classical signature as stated in [Yas96, Thm. 3.1]. Our preference for using [CN20] in the proof of Theorem 4.4 comes from the result being stated in the topological locally flat category. 


\section{Speculation and questions}

5.1. Connection to other conjectures. An alternative approach to the SPC4 is to find a compact 3-manifold $M$ that embeds smoothly in some homotopy 4-sphere $\Sigma^{4}$, but not in $S^{4}$. Notice that if a smooth integral homology sphere $M$ smoothly embeds in $\Sigma$, then $M$ is the boundary of a smooth homology 4-ball [AGL17, Prop. 2.4]. However, there is no known example of a 3-manifold $M$ that is the boundary of a smooth homology 4-ball but that does not embed into $S^{4}$. Both this and the approach in the introduction are hung up at the homological level. Further discussion of knots in homology spheres and concordance in homology cylinders can be found in, for example, HLL18, Dav19.

Corollary 2.5 has some relevance to this which we now discuss (a similar discussion also appears in a comment by Ian Agol on Danny Calegari's blogpost Ago13). The unsolved Schoenflies conjecture proposes that if $\mathcal{S} \subset S^{4}$ is a smoothly embedded submanifold with $\mathcal{S}$ homeomorphic to $S^{3}$, then $\mathcal{S}$ bounds a submanifold $B \subset S^{4}$ that is diffeomorphic to $D^{4}$. The SPC4 implies the Schoenflies conjecture.

Question 5.1. Does every exotic homotopy 4-ball $\mathcal{B}$ smoothly embed into $S^{4}$ ?

Note that if the answer to Question 5.1 is yes, then the Schoenflies conjecture implies the SPC4 and hence the two conjectures are equivalent: If any homotopy 4-ball would embed into $S^{4}$ and thus, by the Schoenflies conjecture, would be diffeomorphic to $D^{4}$, hence all homotopy 4-balls would be standard, so all homotopy 4-spheres would be standard. We have:

Observation 5.2. If the answer to Question 5.1 is yes, then no homotopy 4-ball can have deep slice knots.

Thus by Corollary 2.5, if the answer to Question [5.1] is yes, the approach towards SPC4 mentioned in this section would never succeed. Similarly, there would be no 3-manifold that would smoothly embed into a homotopy 4 -sphere but not into $S^{4}$. This is because any such embedding into a homotopy sphere avoids a standard 4-ball and after removing this ball the complement is a homotopy 4-ball which we assume embeds into $S^{4}$, so this approach to SPC4 would also be a dead end.

\subsection{More questions.}

Question 5.3. Are there any 2-handlebodies $X$ other that $\natural^{k}\left(S^{2} \times B^{2}\right), k \geq 0$, with the property that all $K \subset B^{3} \subset X$ that are slice in $X$ are also slice in $B^{4}$ ? In other words, are there always deep slice local knots when $X \neq \natural^{k}\left(S^{2} \times B^{2}\right)$ ?

One strategy for answering this question would be to start with a framed link $L$ describing a 2-handlebody other than $\natural^{k}\left(S^{2} \times B^{2}\right)$ and to handle-slide $L$ to a new framed link $L^{\prime}$ that contains a knot $K$ that is not slice in $B^{4}$. Then this knot $K$ when considered in a 3-ball $K \subset B^{3} \subset \partial X$ is an example of such a deep slice knot in $X$. This strategy fails to find any non-slice knots $K$ (as it must) for $\natural^{k}\left(S^{2} \times D^{2}\right)$ when we start with $L$ being the 0 -framed unlink - since then all resulting knots $K$ will be ribbon hence slice in $B^{4}$.

In view of the 2-handlebodies constructed in Proposition 4.1 one could ask whether this extension of the Norman-Suzuki trick is the only way to make any knot in the boundary of a manifold bound an embedded disk:

Question 5.4. If $X$ is a 2-handlebody with the property that every knot in the boundary of $X$ is slice in $X$ (no assumption on the relative homology class of 
the disk), does it follow that $X$ decomposes as $X=X_{0} \#\left(S^{2} \times S^{2}\right)$ or $X=$ $X_{0} \#\left(S^{2} \widetilde{\times} S^{2}\right)$ ? More generally, what about the same question without the hypothesis that $X$ be a 2-handlebody?

\section{ACKNOWLEDGMENTS}

The authors would like to thank Anthony Conway, Rob Kirby, Mark Powell, Arunima Ray and Peter Teichner for helpful conversations, their encouragement and guidance. The second author would like to thank Thorben Kastenholz for asking about the decidability of the embedded genus problem in 4-manifolds, which motivated section 4. We are especially grateful to Akira Yasuhara for pointing us to related literature and the work of Suzuki. We are also especially grateful to the anonymous referee for numerous helpful suggestions that improved the exposition. The Max Planck Institute for Mathematics in Bonn supported us financially and with a welcoming research environment.

\section{REFERENCES}

[AGL17] Paolo Aceto, Marco Golla, and Kyle Larson, Embedding 3-manifolds in spin 4manifolds, J. Topol. 10 (2017), no. 2, 301-323, DOI 10.1112/topo.12010. MR3653313

[Ago13] Ian Agol, Comment on the blogpost "Connection between SPC4 and Schoenflies", 2013. https://lamington.wordpress.com/2013/10/18/scharlemann-onschoenflies/, accessed: 2020-03-31.

[Akb10] Selman Akbulut, Cappell-Shaneson homotopy spheres are standard, Ann. of Math. (2) 171 (2010), no. 3, 2171-2175, DOI 10.4007/annals.2010.171.2171. MR2680408

[BN17] Hans U. Boden and Matthias Nagel, Concordance group of virtual knots, Proc. Amer. Math. Soc. 145 (2017), no. 12, 5451-5461, DOI 10.1090/proc/13667. MR 3717970

[Cel18] Daniele Celoria, On concordances in 3-manifolds, J. Topol. 11 (2018), no. 1, 180-200, DOI 10.1112/topo.12051. MR.3784229

[CHH13] Tim D. Cochran, Shelly Harvey, and Peter Horn, Filtering smooth concordance classes of topologically slice knots, Geom. Topol. 17 (2013), no. 4, 2103-2162, DOI 10.2140/gt.2013.17.2103. MR.3109864

[CL86] T. D. Cochran and W. B. R. Lickorish, Unknotting information from 4-manifolds, Trans. Amer. Math. Soc. 297 (1986), no. 1, 125-142, DOI 10.2307/2000460. MR.849471

[CN20] Anthony Conway and Matthias Nagel, Stably slice disks of links, J. Topol. 13 (2020), no. 3, 1261-1301, DOI 10.1112/topo.12154. MR4125756

[Con19] Anthony Conway, The Levine-Tristram signature: a survey, 2019. arXiv:1903.04477.

[COT03] Tim D. Cochran, Kent E. Orr, and Peter Teichner, Knot concordance, Whitney towers and $L^{2}$-signatures, Ann. of Math. (2), 157(2):433-519, 2003. https://doi.org/10. 4007/annals.2003.157.433, arXiv:math/9908117

[Dav19] Christopher W. Davis, Concordance, crossing changes, and knots in homology spheres, Canad. Math. Bull. 63 (2020), no. 4, 744-754, DOI 10.4153/s0008439519000791. MR4176766

[DNPR18] Christopher W. Davis, Matthias Nagel, JungHwan Park, and Arunima Ray, Concordance of knots in $S^{1} \times S^{2}$, J. Lond. Math. Soc. (2) 98 (2018), no. 1, 59-84, DOI 10.1112/jlms.12125. MR 3847232

[FGMW10] Michael Freedman, Robert Gompf, Scott Morrison, and Kevin Walker, Man and machine thinking about the smooth 4-dimensional Poincaré conjecture, Quantum Topol. 1 (2010), no. 2, 171-208, DOI 10.4171/QT/5. MR2657647

[FNOP19] Stefan Friedl, Matthias Nagel, Patrick Orson, and Mark Powell, Satellites and concordance of knots in 3-manifolds, Trans. Amer. Math. Soc. 371 (2019), no. 4, 2279-2306, DOI 10.1090/tran/7313. MR3896081

[FQ90] Michael H. Freedman and Frank Quinn, Topology of 4-manifolds, Princeton Mathematical Series, vol. 39, Princeton University Press, Princeton, NJ, 1990. MR.1201584

[Fre82] Michael Hartley Freedman, The topology of four-dimensional manifolds, J. Differential Geometry 17 (1982), no. 3, 357-453. MR679066 
[Gil81] Patrick M. Gilmer, Configurations of surfaces in 4-manifolds, Trans. Amer. Math. Soc. 264 (1981), no. 2, 353-380, DOI 10.2307/1998544. MR603768

[HLL18] Jennifer Hom, Adam Simon Levine, and Tye Lidman, Knot concordance in homology cobordisms, 2018. arXiv: 1801.07770

[Kam17] Seiichi Kamada, Surface-knots in 4-space: An introduction, Springer Monographs in Mathematics, Springer, Singapore, 2017, DOI 10.1007/978-981-10-4091-7. MR.3588325

[Kir95] Rob Kirby (ed.), Problems in low-dimensional topology, Geometric topology (Athens, GA, 1993), AMS/IP Stud. Adv. Math., vol. 2, Amer. Math. Soc., Providence, RI, 1997, pp. 35-473. MR1470751

[KM13] P. B. Kronheimer and T. S. Mrowka, Gauge theory and Rasmussen's invariant, J. Topol. 6 (2013), no. 3, 659-674, DOI 10.1112/jtopol/jtt008. MR3100886

[KSS82] Akio Kawauchi, Tetsuo Shibuya, and Shin'ichi Suzuki, Descriptions on surfaces in four-space. I. Normal forms, Math. Sem. Notes Kobe Univ. 10 (1982), no. 1, 75-125. MR672939

[Lev69] J. Levine, Invariants of knot cobordism, Invent. Math. 8 (1969), 98-110; addendum, ibid. 8 (1969), 355, DOI 10.1007/BF01404613. MR253348

[Lic97] W. B. Raymond Lickorish, An introduction to knot theory, Graduate Texts in Mathematics, vol. 175, Springer-Verlag, New York, 1997, DOI 10.1007/978-1-4612-0691-0. MR.1472978

[Mil65] John Milnor, Lectures on the h-cobordism theorem: Notes by L. Siebenmann and J. Sondow, Princeton University Press, Princeton, N.J., 1965. MR0190942

[NOPP19] Matthias Nagel, Patrick Orson, JungHwan Park, and Mark Powell, Smooth and topological almost concordance, Int. Math. Res. Not. IMRN 23 (2019), 7324-7355, DOI 10.1093/imrn/rnx338. MR4039014

[Nor69] R. A. Norman, Dehn's lemma for certain 4-manifolds, Invent. Math. 7 (1969), 143147, DOI 10.1007/BF01389797. MR246309

[Per03] Grisha Perelman, Finite extinction time for the solutions to the Ricci flow on certain three-manifolds, 2003. arXiv:math/0307245

[Roh71] V. A. Rohlin, Two-dimensional submanifolds of four-dimensional manifolds (Russian), Funkcional. Anal. i Priložen. 5 (1971), no. 1, 48-60. MR0298684

[Sch03] Rob Schneiderman, Algebraic linking numbers of knots in 3-manifolds, Algebr. Geom. Topol. 3 (2003), 921-968, DOI 10.2140/agt.2003.3.921. MR2012959

[Suz69] Shin'ichi Suzuki, Local knots of 2-spheres in 4-manifolds, Proc. Japan Acad. 45 (1969), 34-38. MR248851

[Tri69] A. G. Tristram, Some cobordism invariants for links, Proc. Cambridge Philos. Soc. 66 (1969), 251-264, DOI 10.1017/s0305004100044947. MR248854

[Wal99] C. T. C. Wall, Surgery on compact manifolds, 2nd ed., Mathematical Surveys and Monographs, vol. 69, American Mathematical Society, Providence, RI, 1999. Edited and with a foreword by A. A. Ranicki, DOI 10.1090/surv/069. MR1687388

[Yas91] Akira Yasuhara, $(2,15)$-torus knot is not slice in $\mathbf{C P}^{2}$, Proc. Japan Acad. Ser. A Math. Sci. 67 (1991), no. 10, 353-355. MR 1151354

[Yas92] Akira Yasuhara, On slice knots in the complex projective plane, Rev. Mat. Univ. Complut. Madrid 5 (1992), no. 2-3, 255-276. MR.1195083

[Yas96] Akira Yasuhara, Connecting lemmas and representing homology classes of simply connected 4-manifolds, Tokyo J. Math. 19 (1996), no. 1, 245-261, DOI 10.3836/tjm/1270043232. MR/1391941

[Yil18] Eylem Zeliha Yildiz, A note on knot concordance, Algebr. Geom. Topol. 18 (2018), no. 5, 3119-3128, DOI 10.2140/agt.2018.18.3119. MR3848410

Department of Mathematics, University of California, Berkeley, Berkeley, CaliFORNIA, 94720-3840

Email address: michael.r.klug@gmail.com

URL: https://math.berkeley.edu/ mrklug/

Max-Planck-Institut für Mathematik, Bonn, Germany

Email address: bruppik@mpim-bonn.mpg.de

URL: https://ben300694.github.io/ 